\title{
Motor Control Theories: Providing an Integrated Structural Model Based on Common Concepts
}

\section{Mostafa Teymuri Kheravi ${ }^{1}$, Alireza Saberi Kakhki", Mohammad Darainy ${ }^{3}$, Sara Sarhadi ${ }^{4}$, Zeynab Rastegar ${ }^{2}$}

${ }^{1}$ Bojnourd Branch, Islamic Azad University, Bojnourd, Iran

${ }^{2}$ Department of Motor Behavior, Ferdowsi University of Mashhad, Mashhad, Iran

${ }^{4}$ Department of Neuromuscular, Shahid Rajaee Teacher Training University, Tehran, Iran

${ }^{3}$ Department of Psychology, McGill University, Montreal, Canada

\section{Article Info:}

\section{ABSTRACT}

Introduction: Several investigations on the mechanism of motor control and learning leads to multiple theories in this field. The purpose of this study was to examine these theories and integrate them into a conceptual model for a better understanding of motor control and learning. Conclusion: A series of motor control studies have demonstrated that many movements, especially reaching movement that requires high final position accuracy, consist of two acceleration and deceleration phases. Review of some motor control theories show that they are consist of two parts so that each one controls a particular part of the reaching movement by different mechanisms. Integrating these theories, based on the neural structures involved at each stage, provides a comprehensive understanding of how to control the movement. We suggest conceptual integrated model.

*Corresponding Author: Alireza Saberi Kakhki

E-mail: Askakhki@um.ac.ir 


\section{نظريه هاى كنترل حركتى: ارائهُ يك مدل ساختارى يكيار جه بر اساس مفاهيم مشترك}

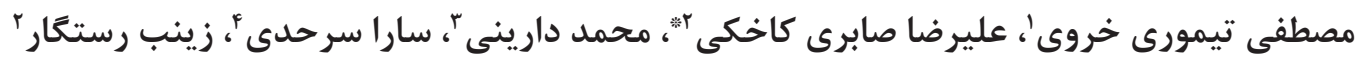

$$
\begin{aligned}
& \text { 'واحد بجنورد، دانشخاه آزاد اسلامى، بجنورد، ايران }
\end{aligned}
$$

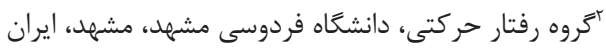

$$
\begin{aligned}
& \text { "تروه روانشناسى، دانشعاه مك تَيل، مونترال، كانادا }
\end{aligned}
$$

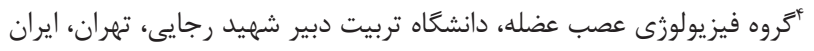

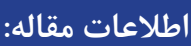

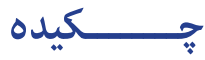

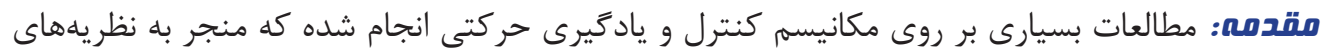

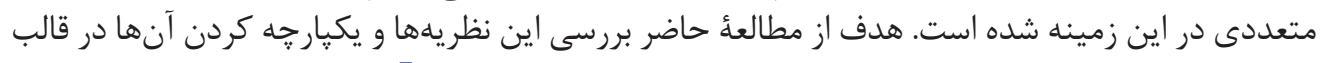

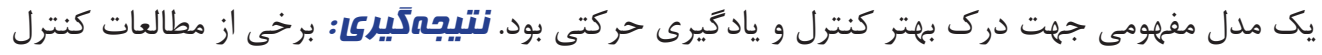

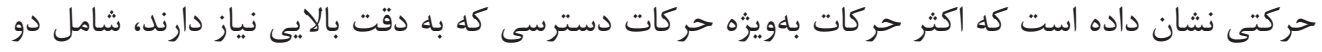

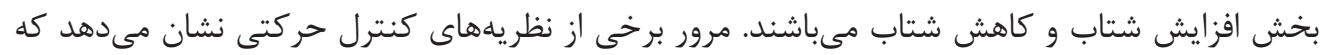

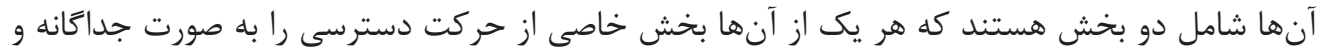

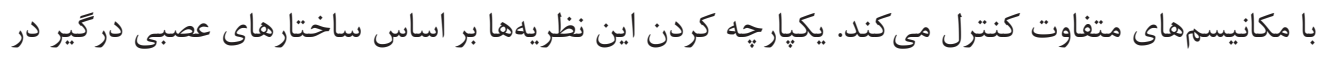

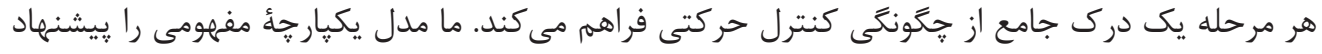
مى كنيم.

كليد وازهها:

ا مغز

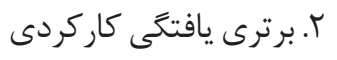

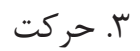

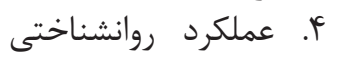
حركتى 
حالتهاى كنتر لى بيشخور اندى و بازخور دى

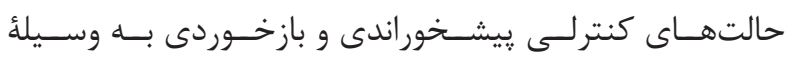

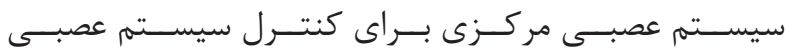

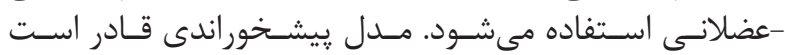

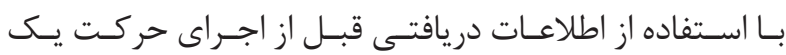

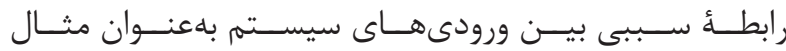

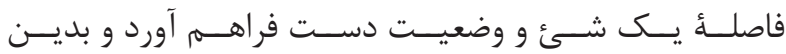

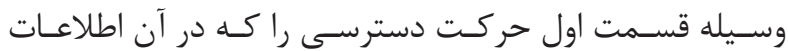

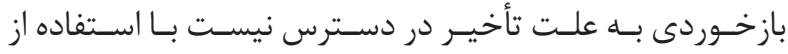

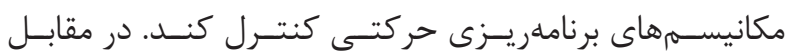

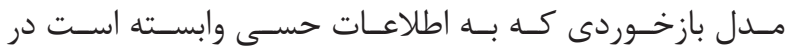

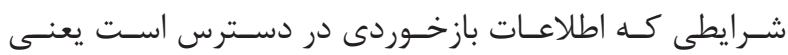

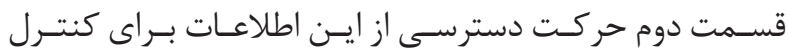

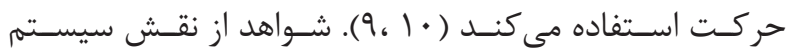

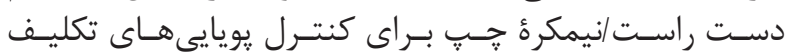

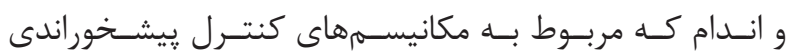
و نقـش سيسـتم دسـت جِ

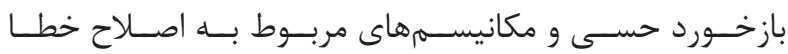

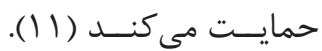

كنترل حلقه باز و كنترل حلقه بسته

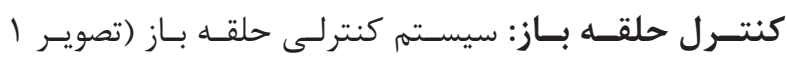

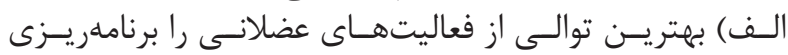

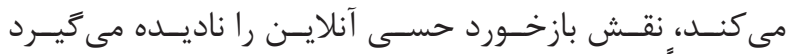

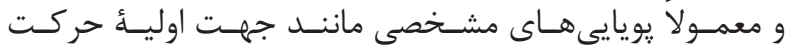

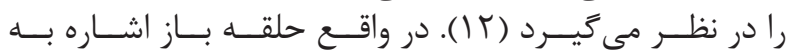

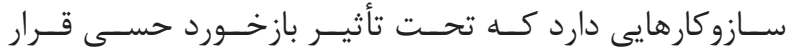

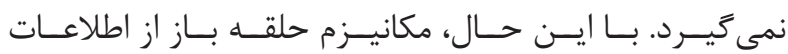

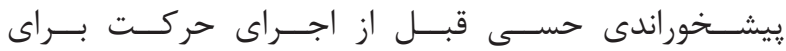

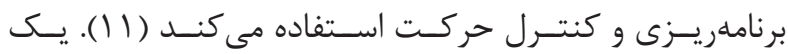

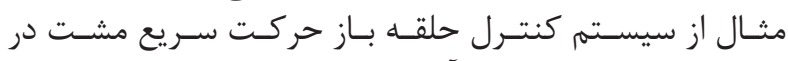

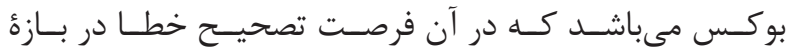

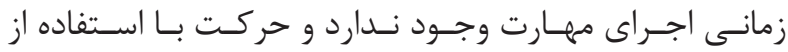

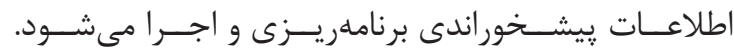

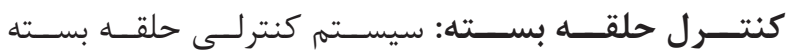

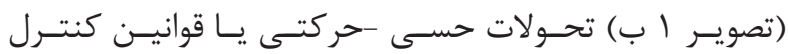

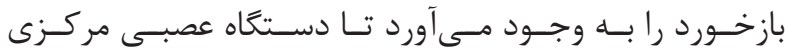

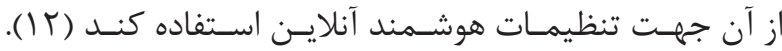

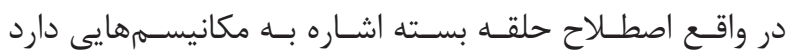

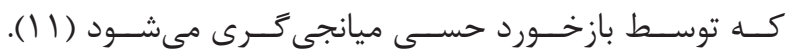

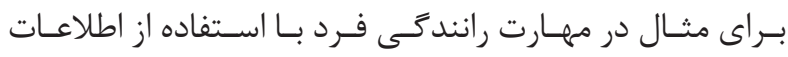

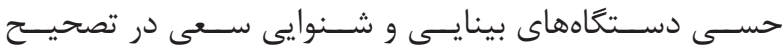

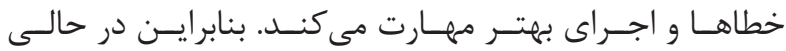

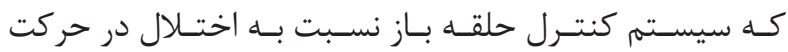

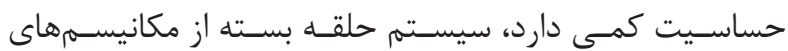

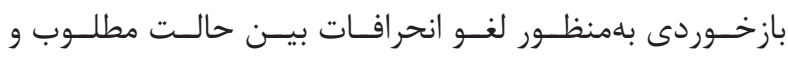
واقعـى بــدن اســتفاده مى كنــد (ب).

${ }^{1}$ Feedforward
كنتــــ حركتـى فراينـدى اسـت كـهـ در آن انســانها و حيوانات

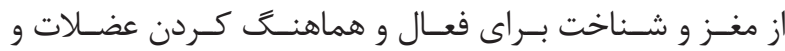

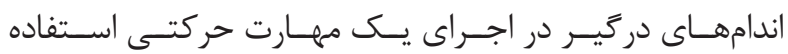

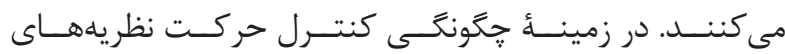

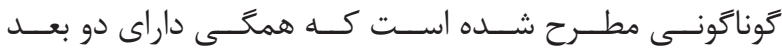

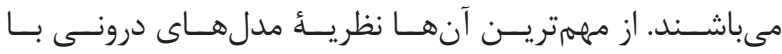

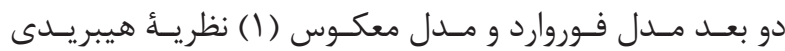

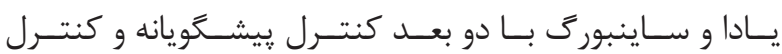

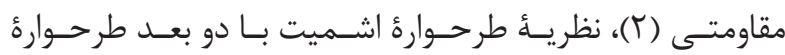

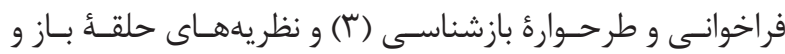

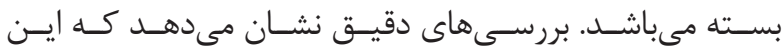

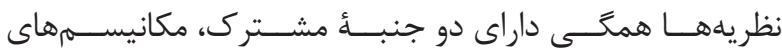

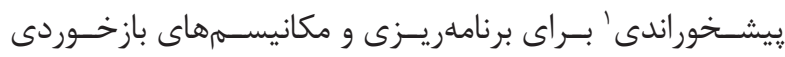

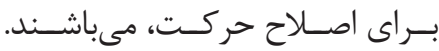

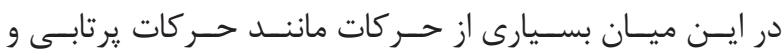

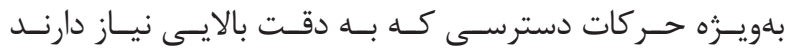

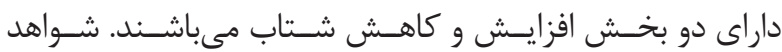

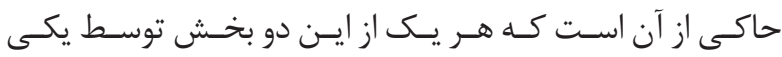

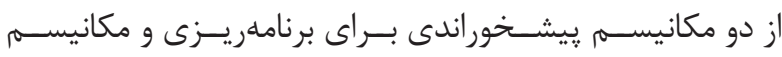

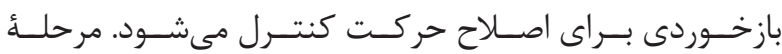

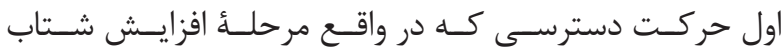

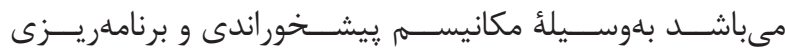

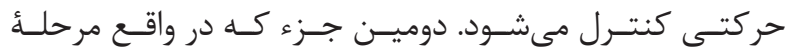

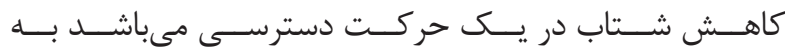

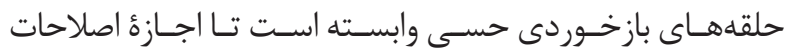

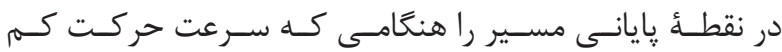

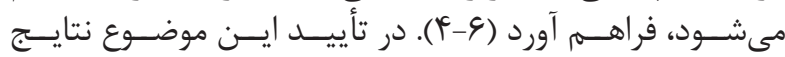

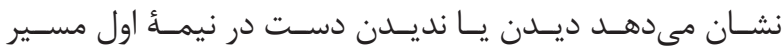

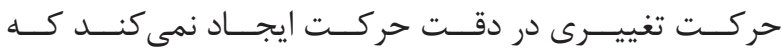

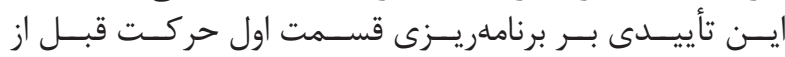

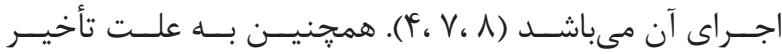

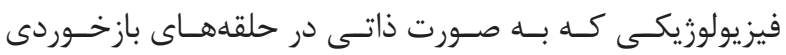

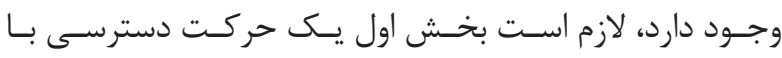

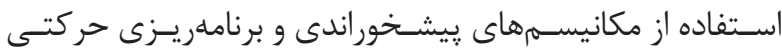

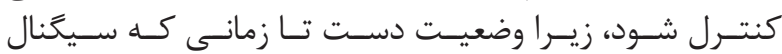

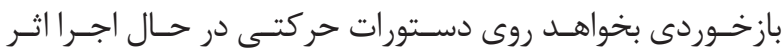

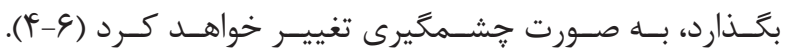

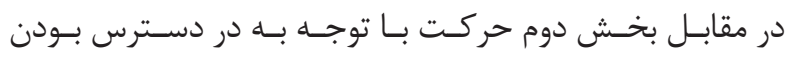

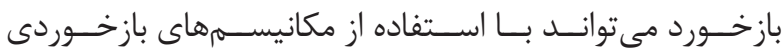

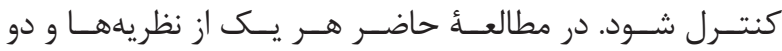

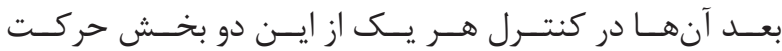

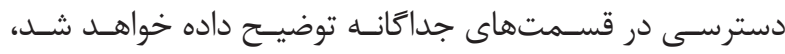

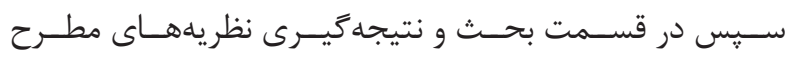

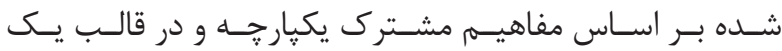

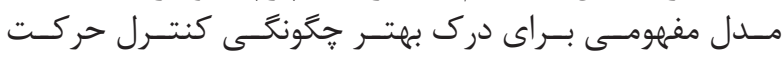

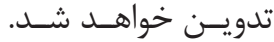




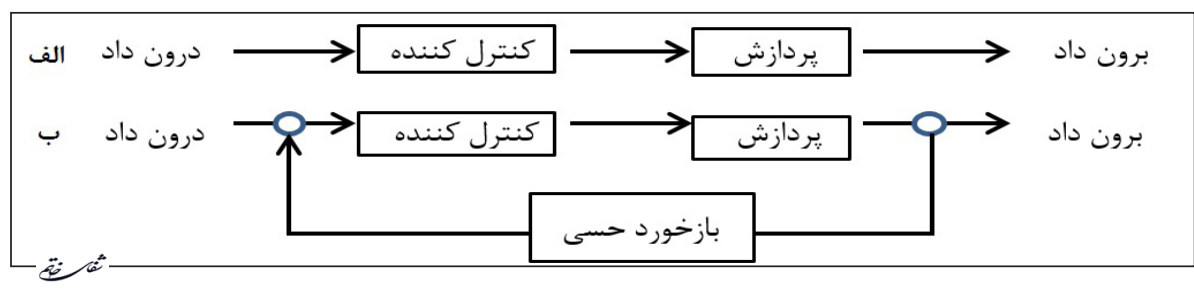

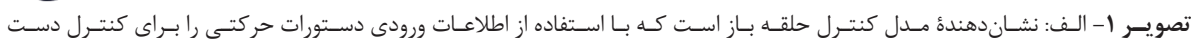

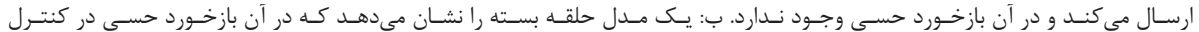

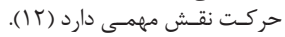

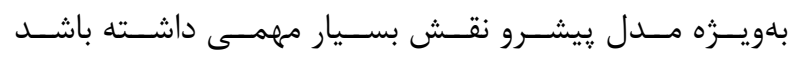
( $(1 \vee-19)$

$$
\text { مدل درونى معكوس }
$$

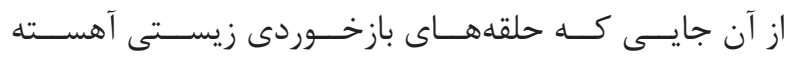

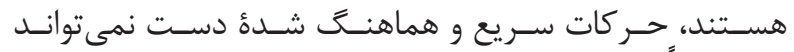

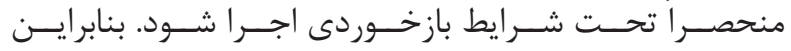

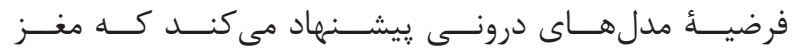

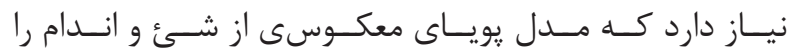

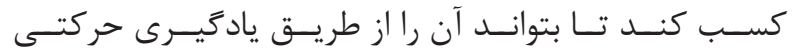

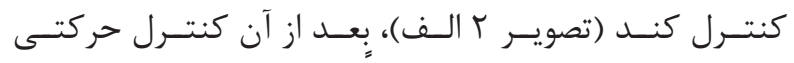

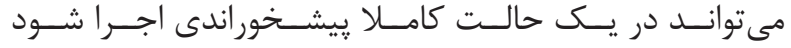

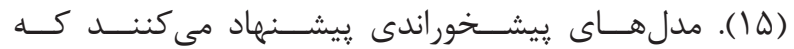

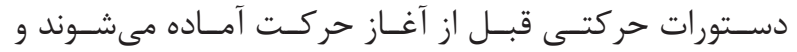

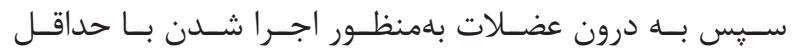

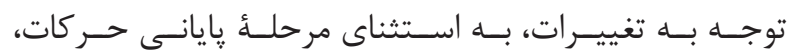

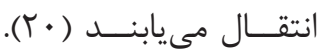

$$
\text { مدل درونى بيشرو }
$$

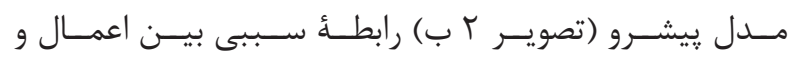

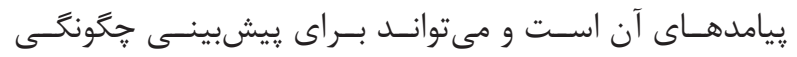

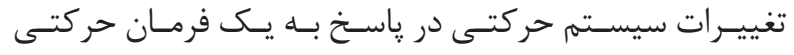

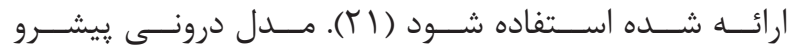

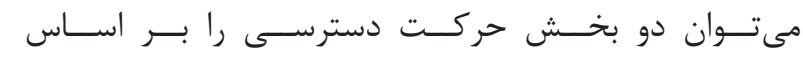

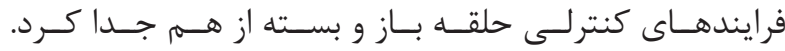

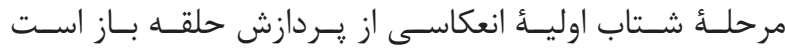

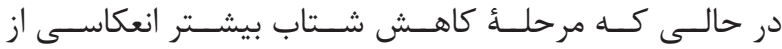

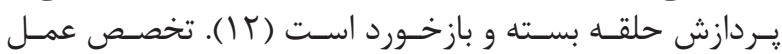

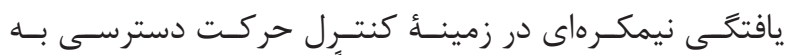

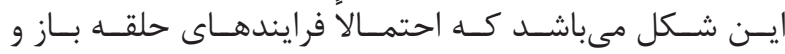

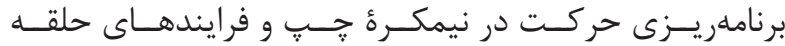

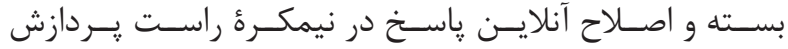

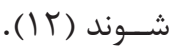

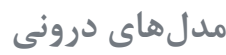

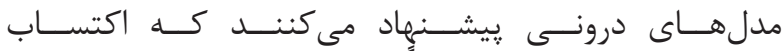

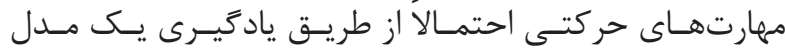

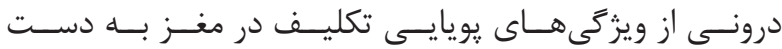

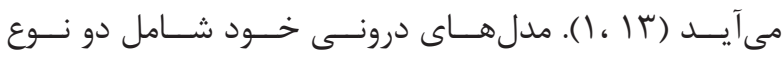

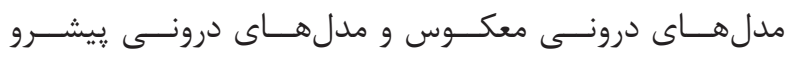

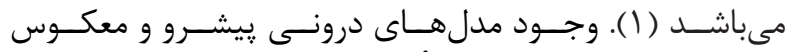

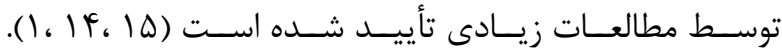

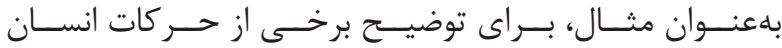

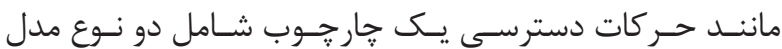

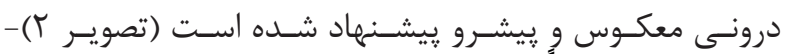

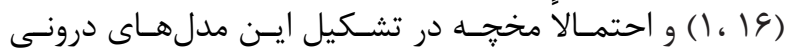

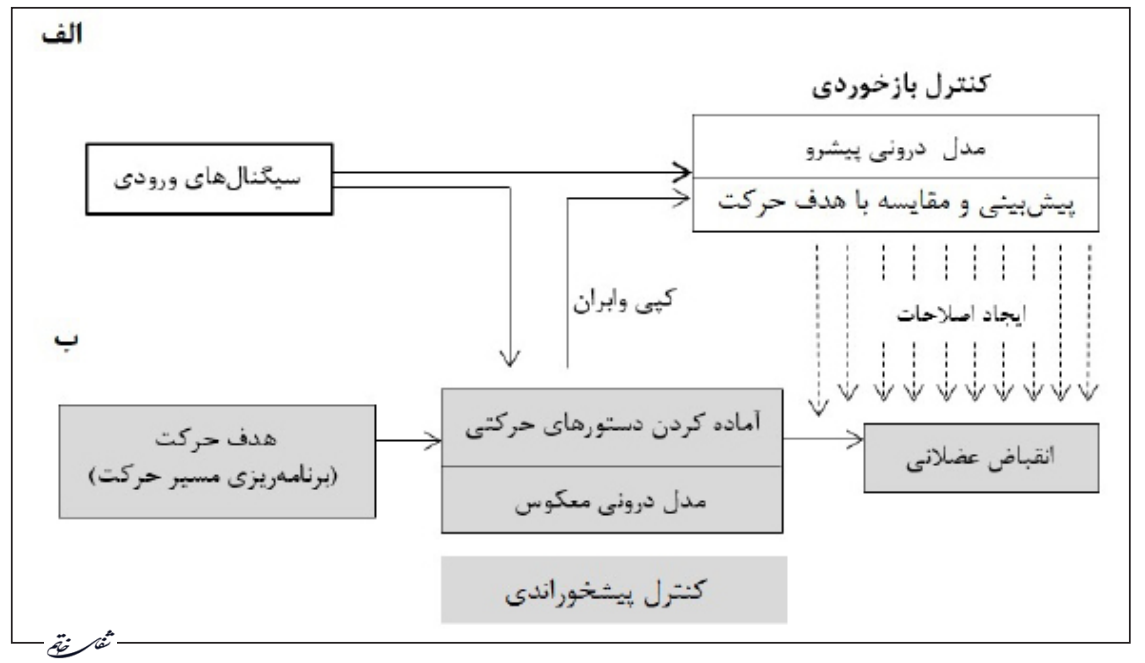

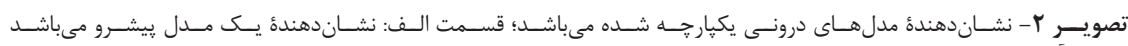

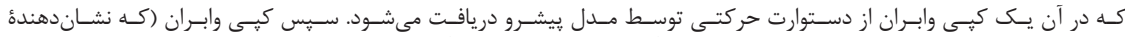

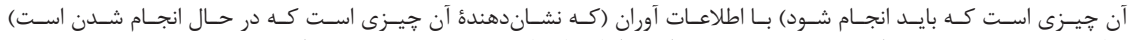

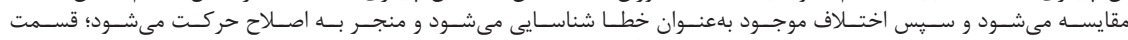

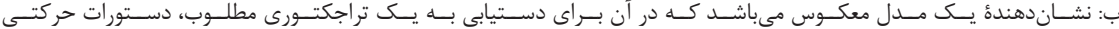

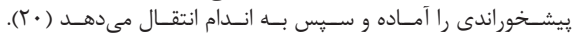




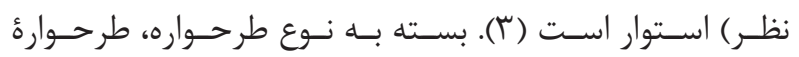

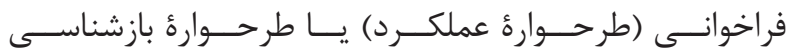

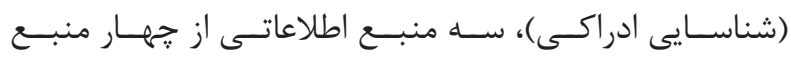

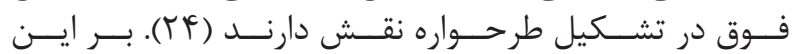

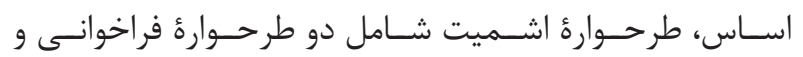

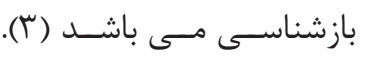

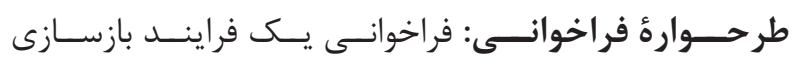

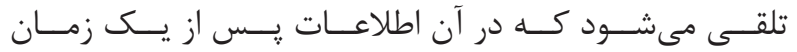

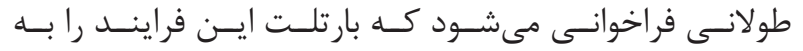

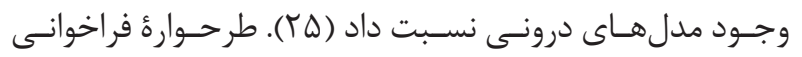

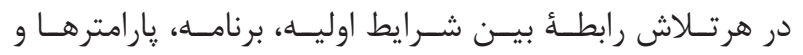

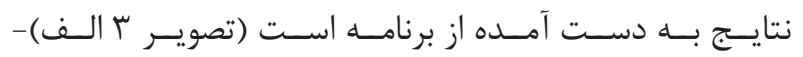

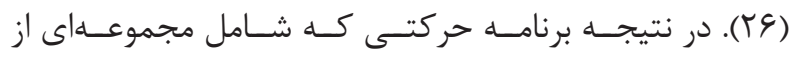

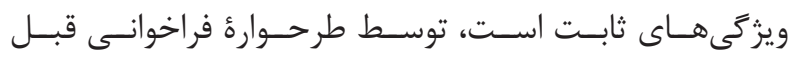

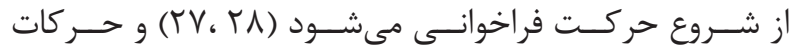

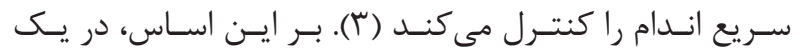

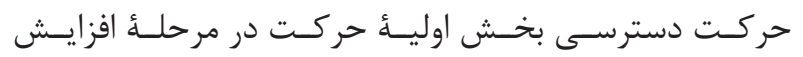

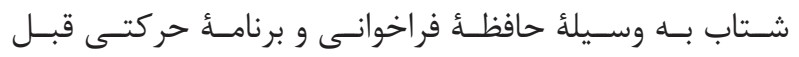

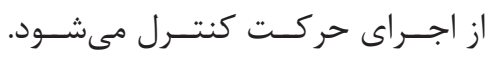

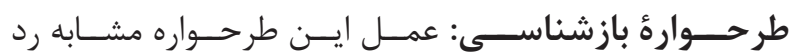

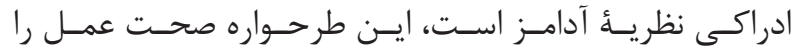

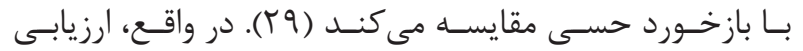

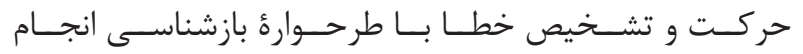

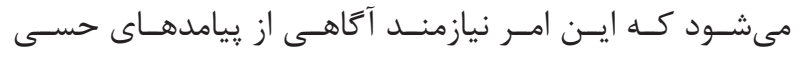

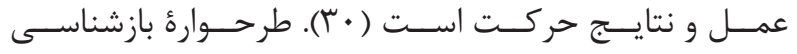

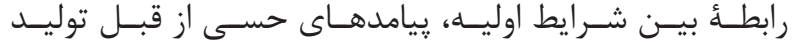

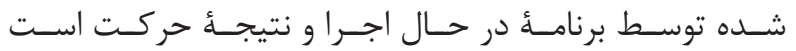

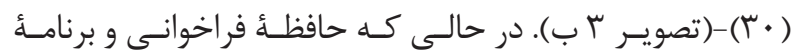

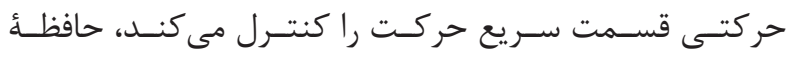

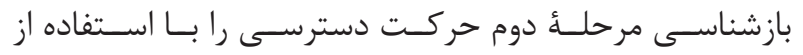

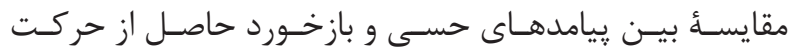

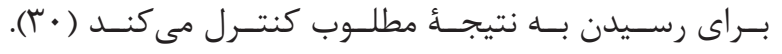

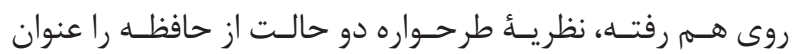

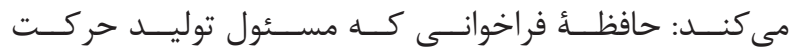

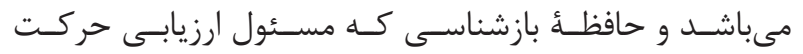

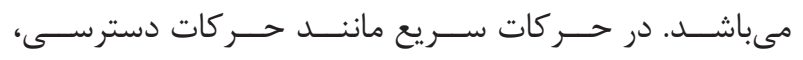

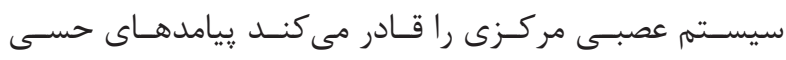

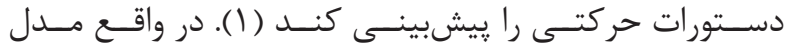

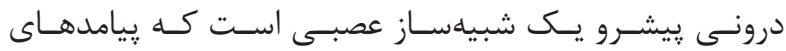

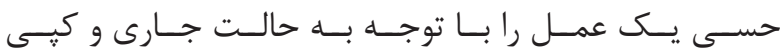

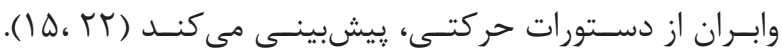

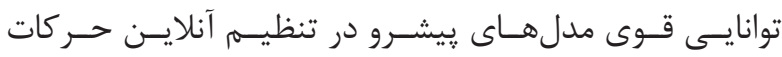

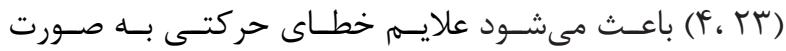

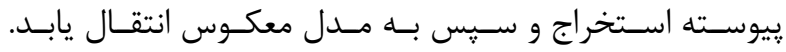

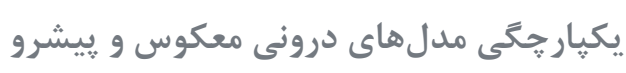

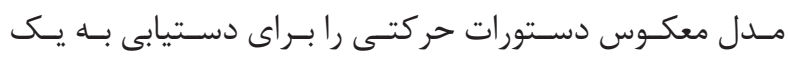

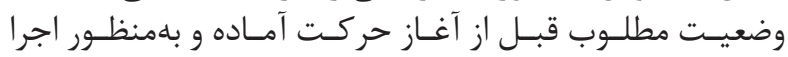

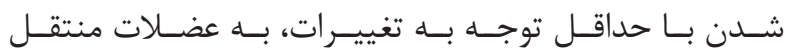

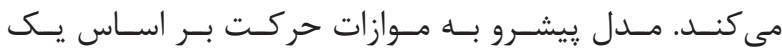

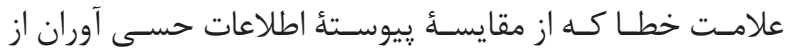

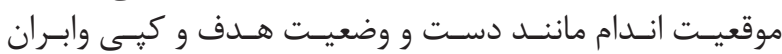

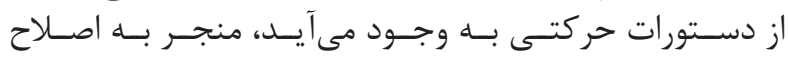

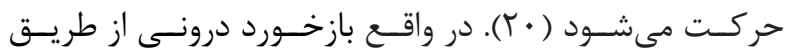

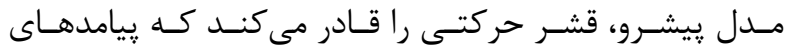

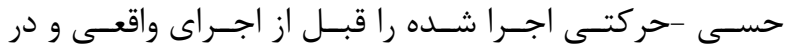

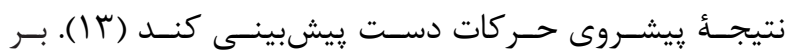

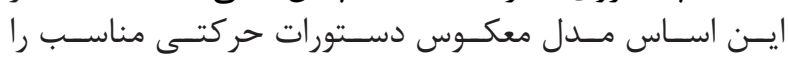

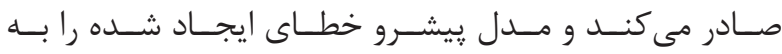

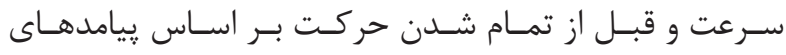

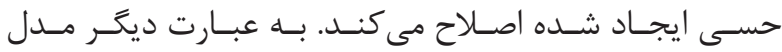

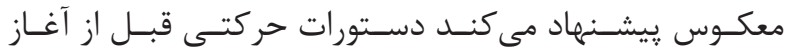

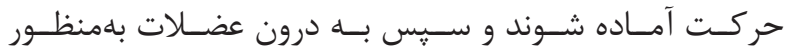

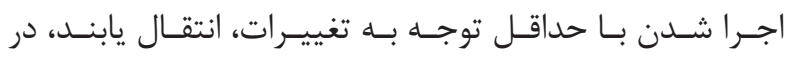

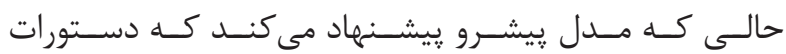

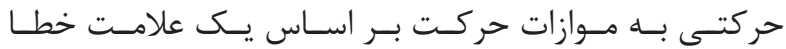

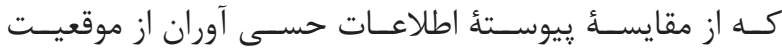

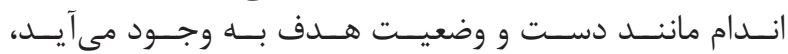

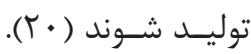

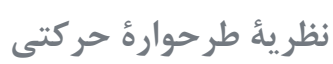

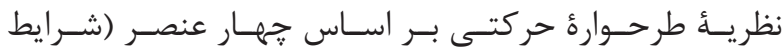

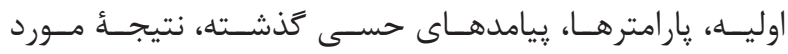

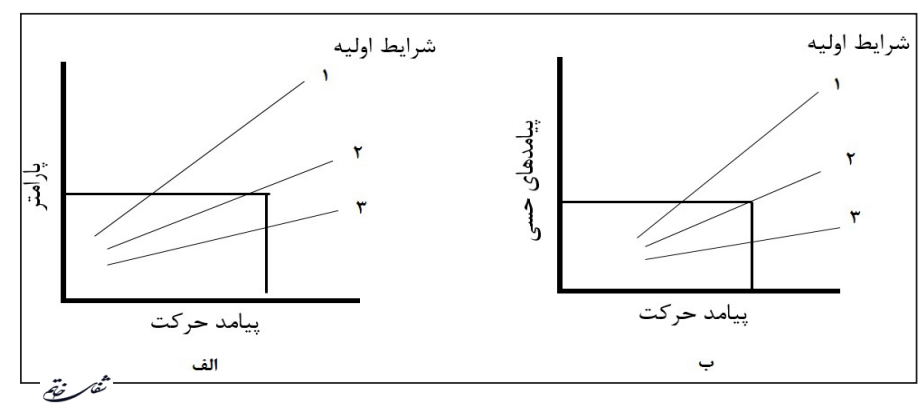

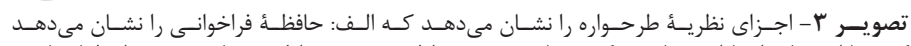

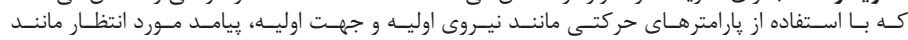

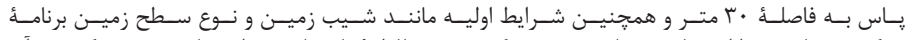

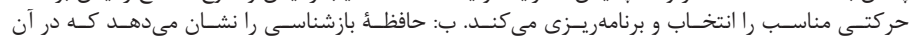

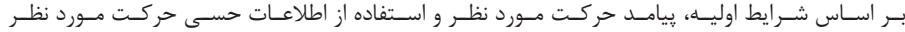




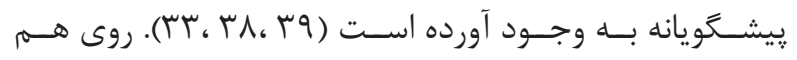

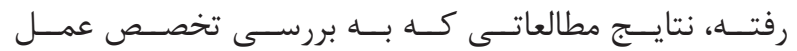

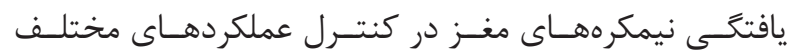

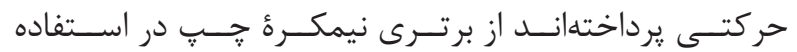

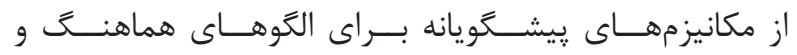

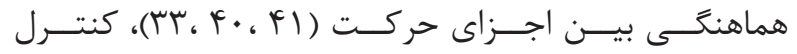

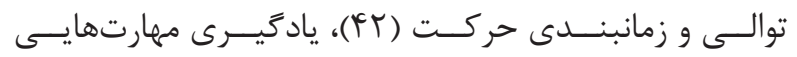

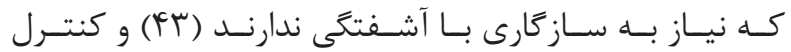

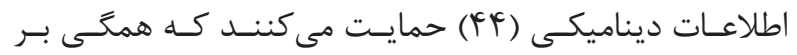

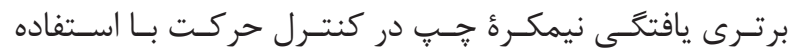

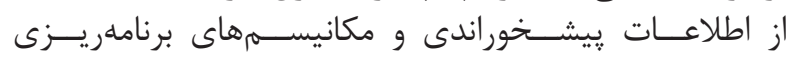

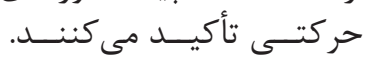

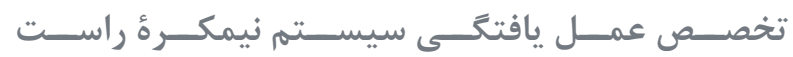

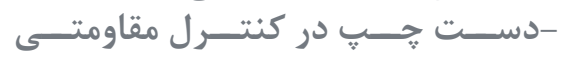

ممكـن اسـت سيسـتم نيمكـرؤ جـــ

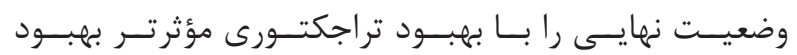

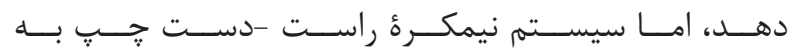

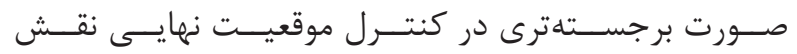

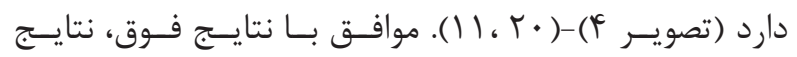

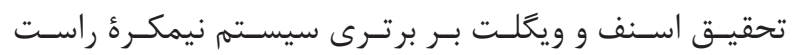
-دسـت جــ

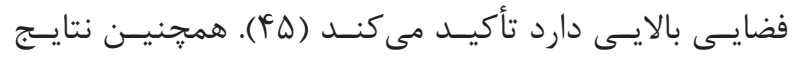

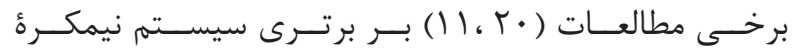

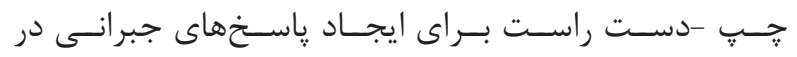

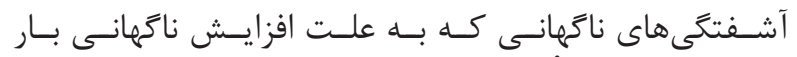

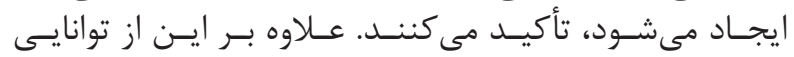

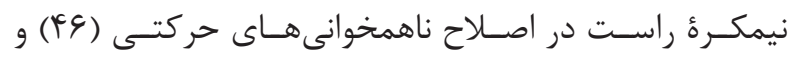

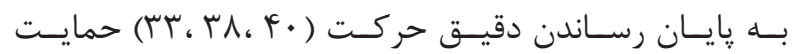

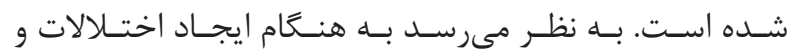

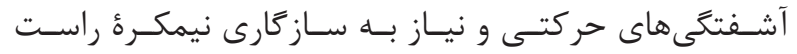

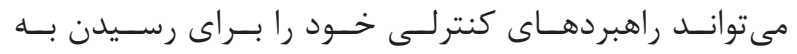

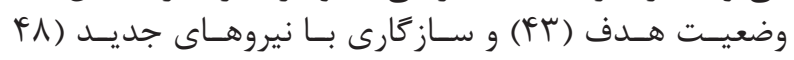
Sر (FV،

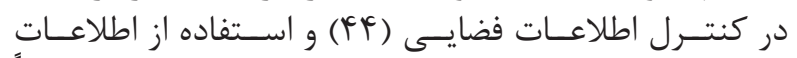

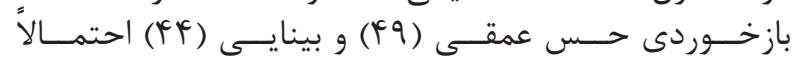

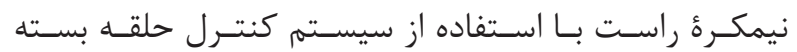

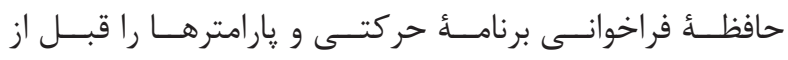

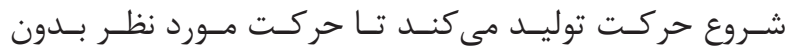

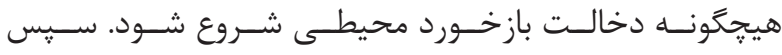

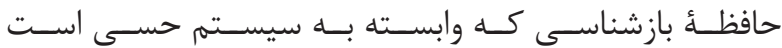

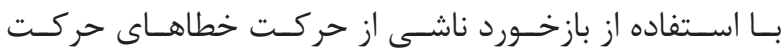

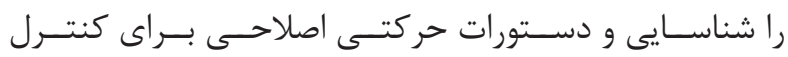

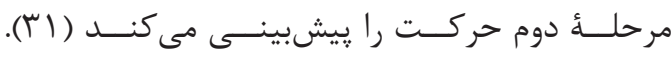

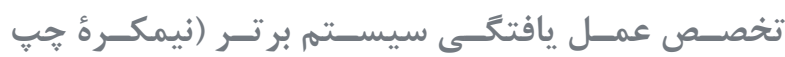

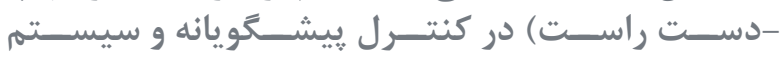

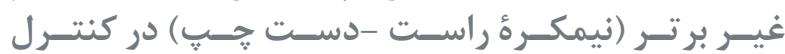
مقاومتــى حركست

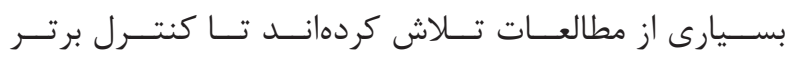

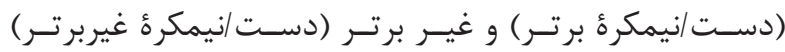

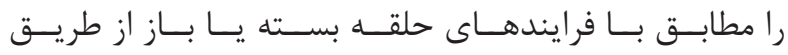

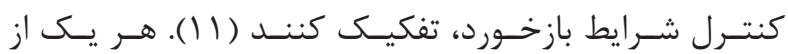

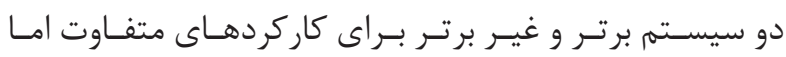

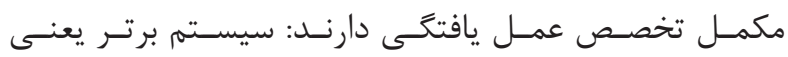

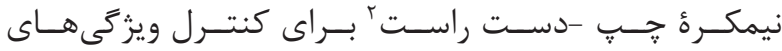

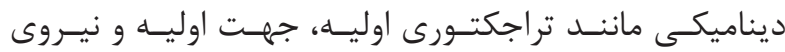

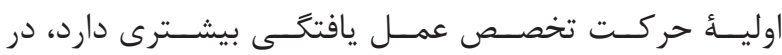

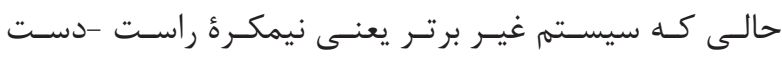

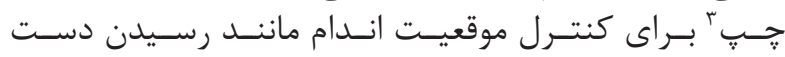

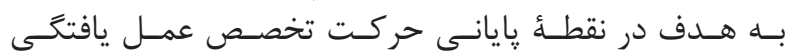

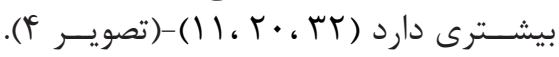

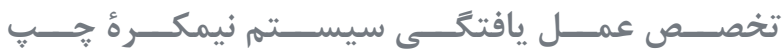

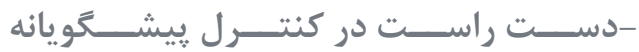

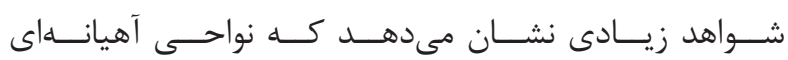

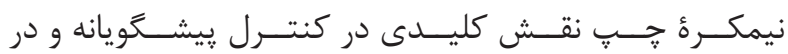

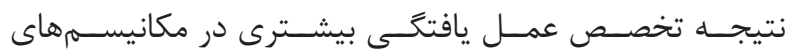

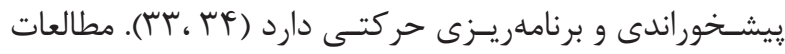

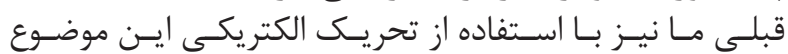

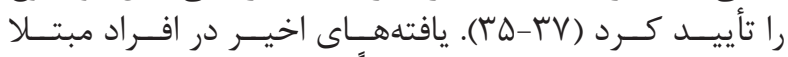

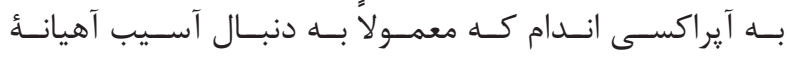
جــ

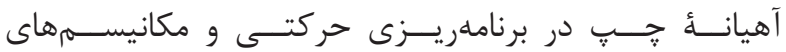

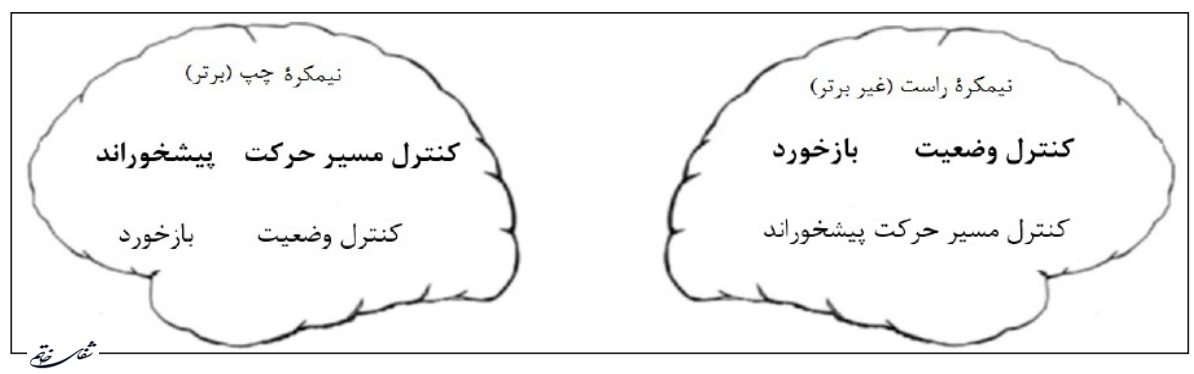

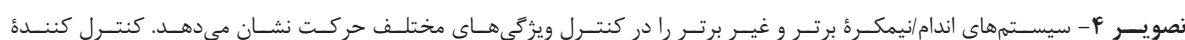

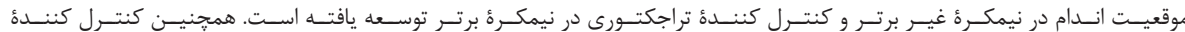

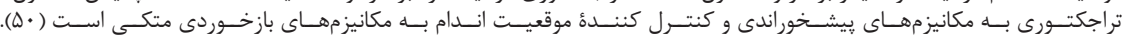




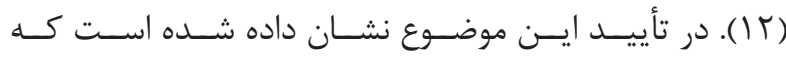
آسـيب بــه نيمكـرهٔ جــ

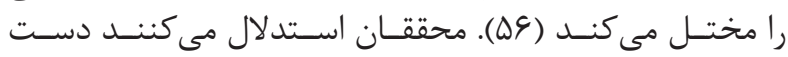

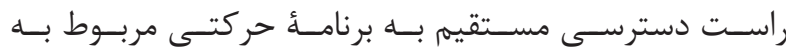

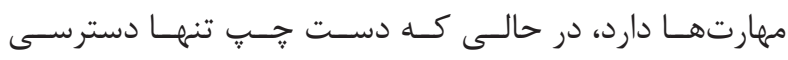

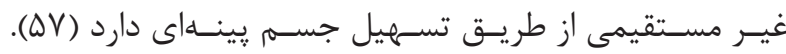

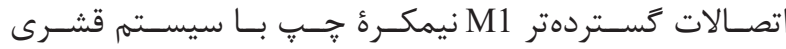

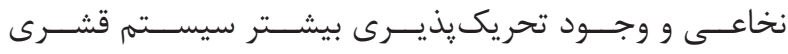

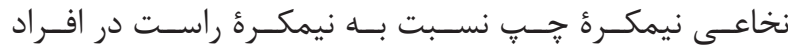

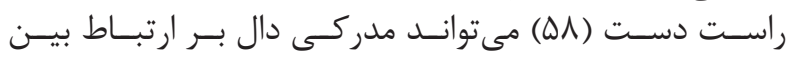

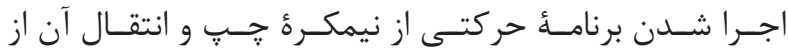

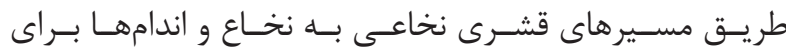

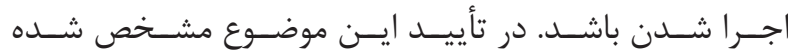

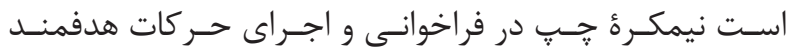

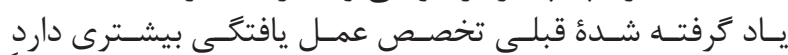

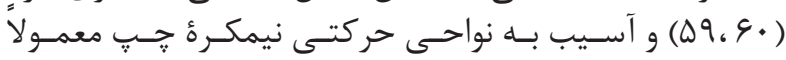

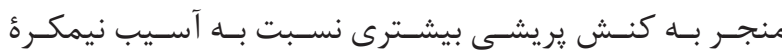

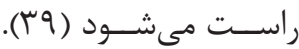

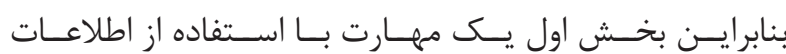

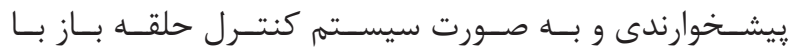

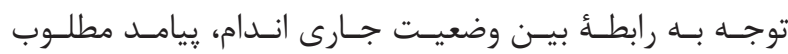

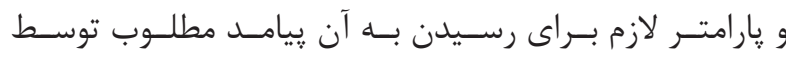

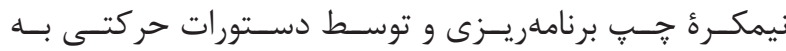

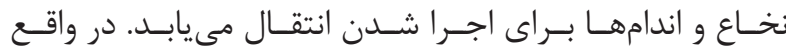

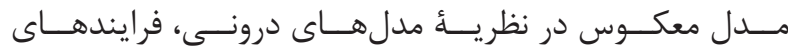

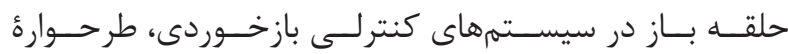

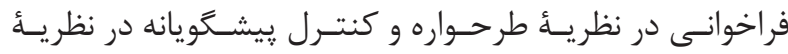

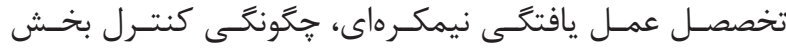

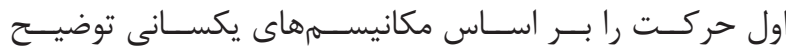

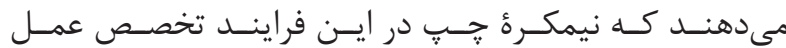

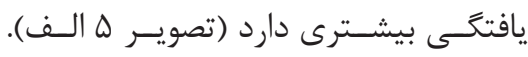

و اسـتفاده از بازخــورد حسـى در رسـيدن دسـت بـهـ هـدف

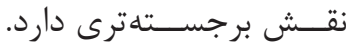

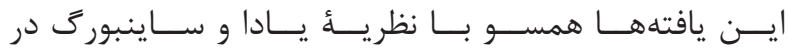

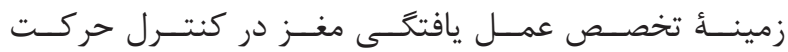

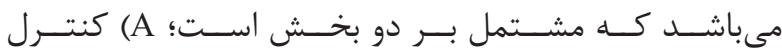

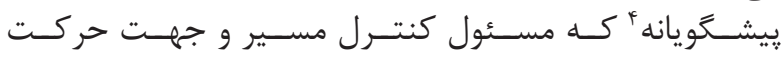

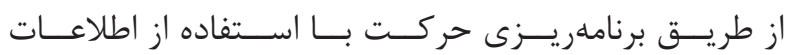

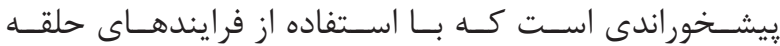

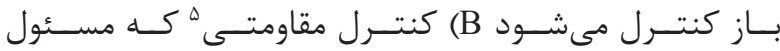

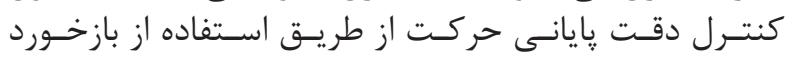

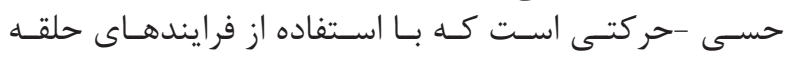

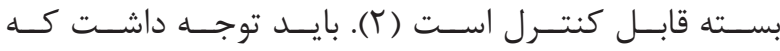

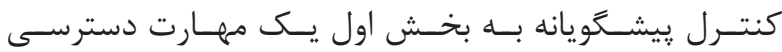

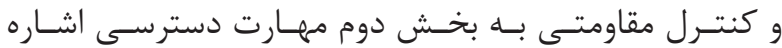
دار نتيجه

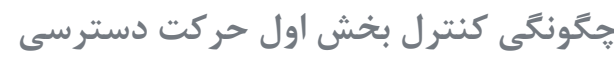

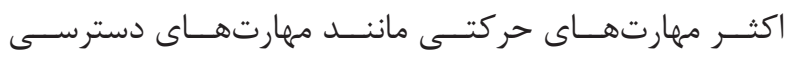

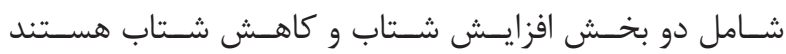

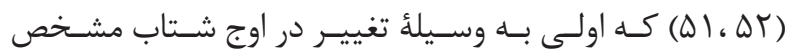

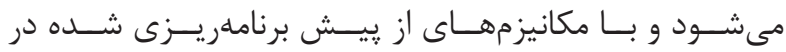

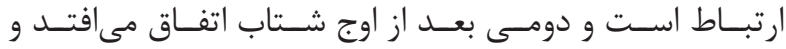

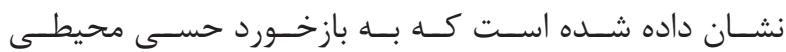

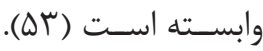

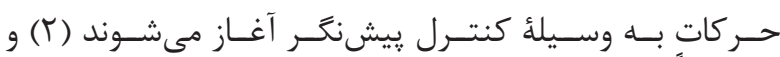

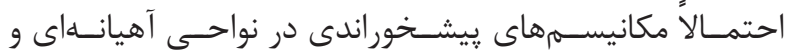

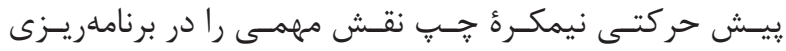

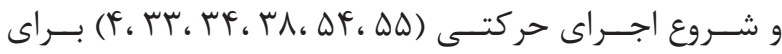

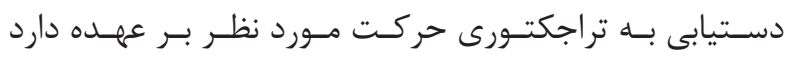

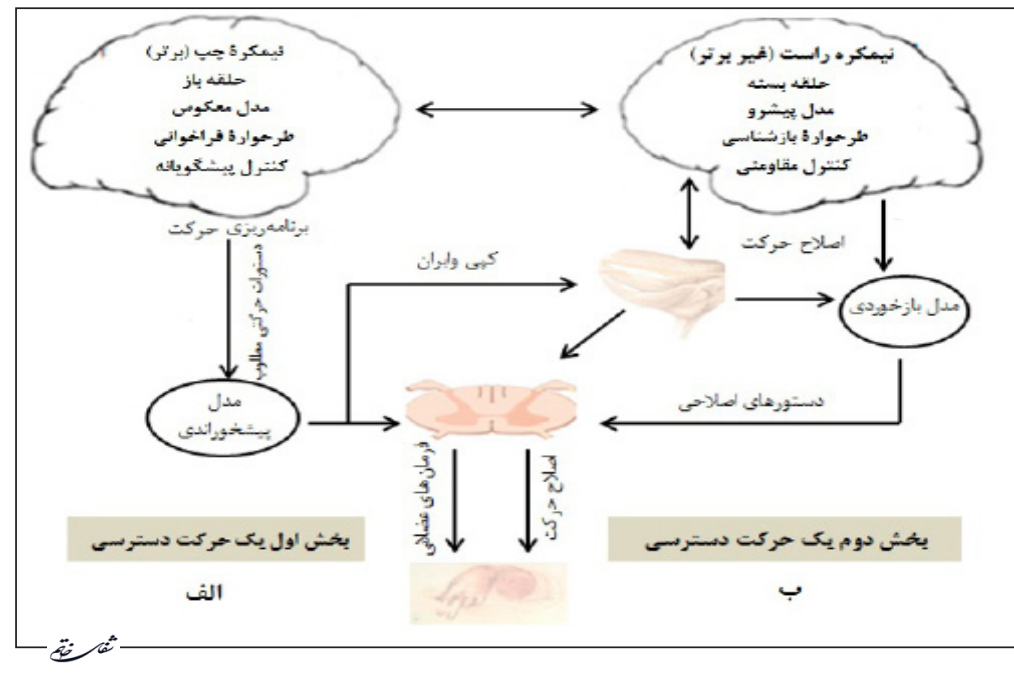

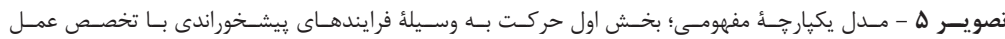

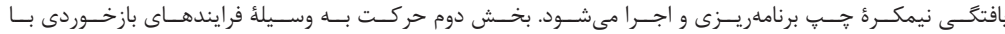

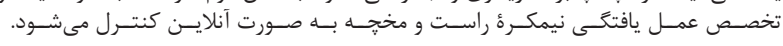




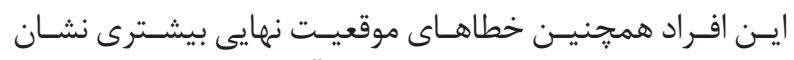

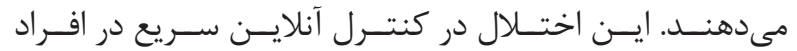

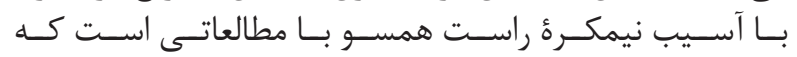

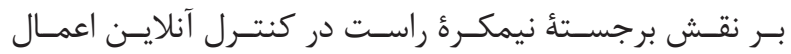

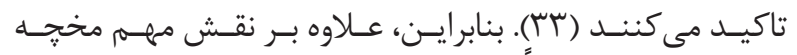

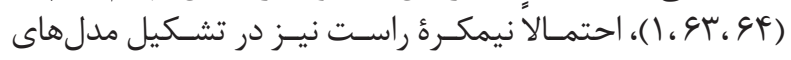

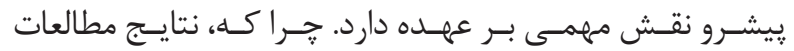

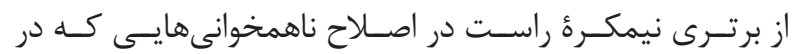

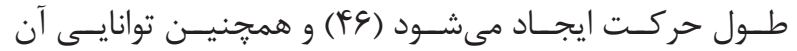

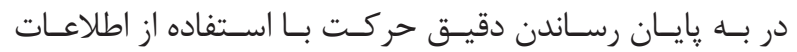

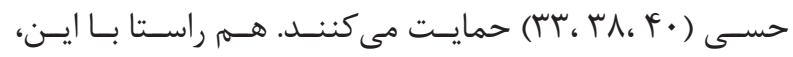

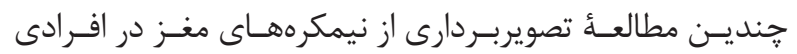

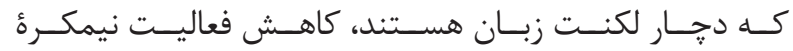

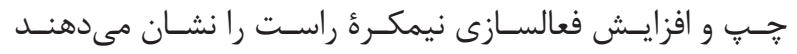

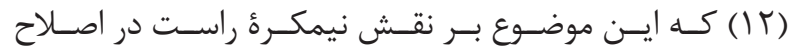

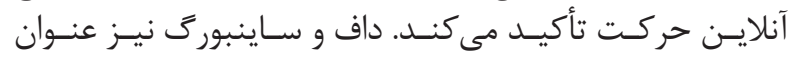

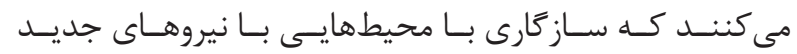

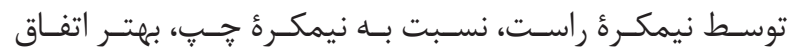

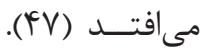

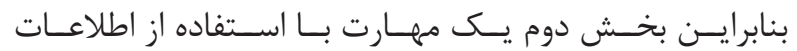

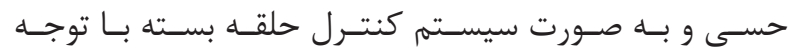

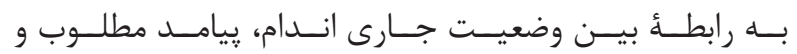

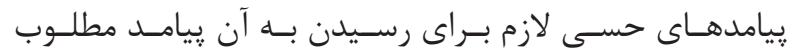

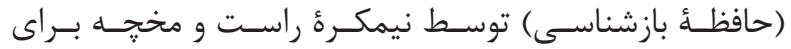

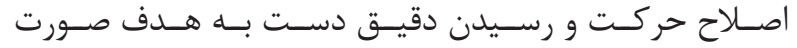

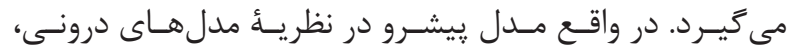

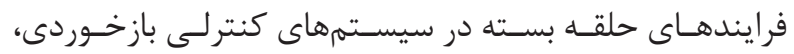

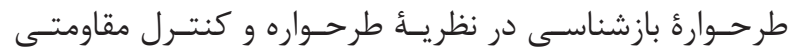

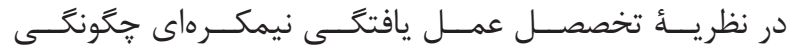

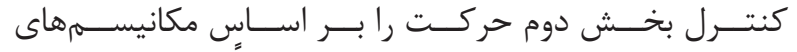

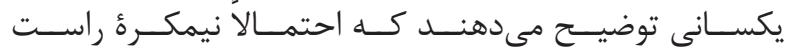

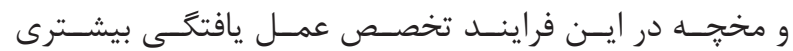

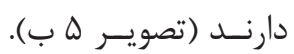

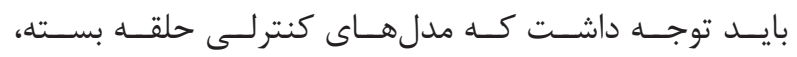

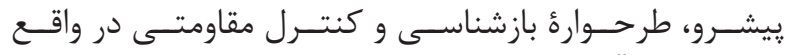

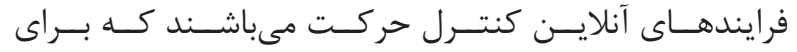

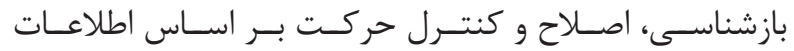

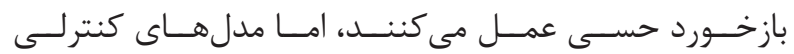

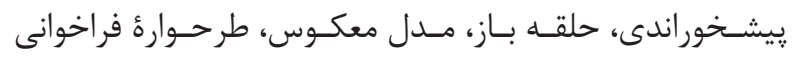

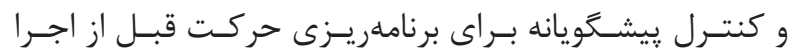

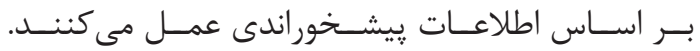

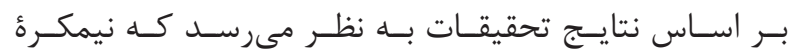

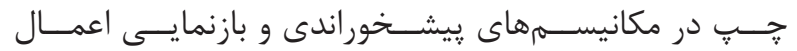

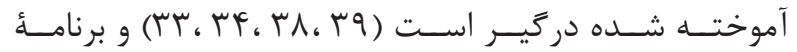

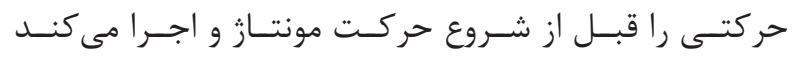

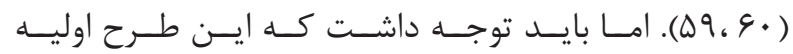

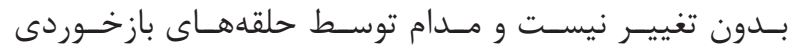

\section{جَحَّنَى كنترل بخش دوم حركت دسترسى}

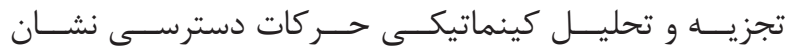

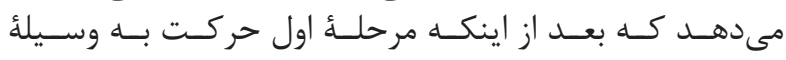

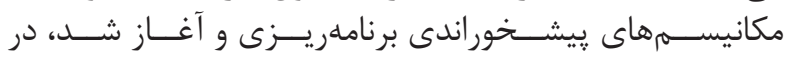

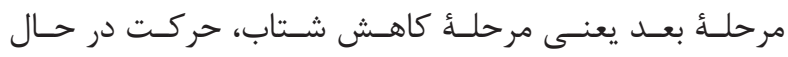

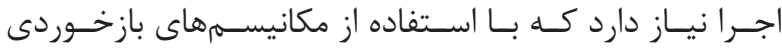

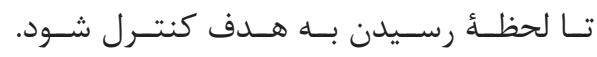

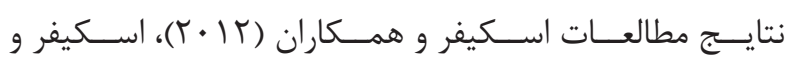

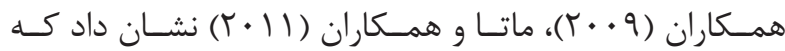

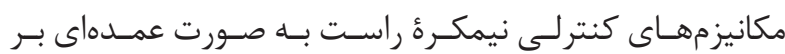

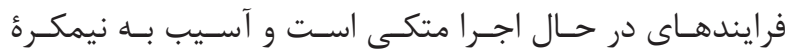

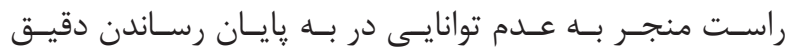

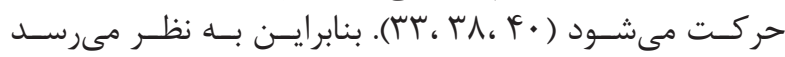

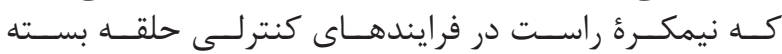

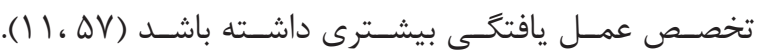

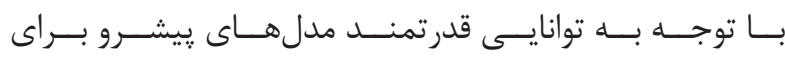

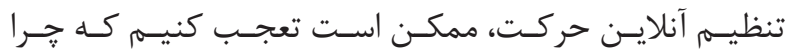

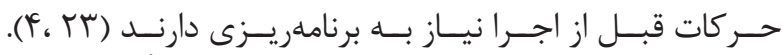

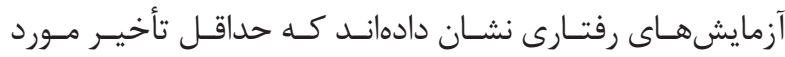

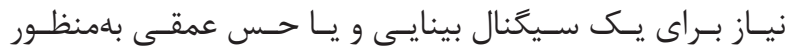

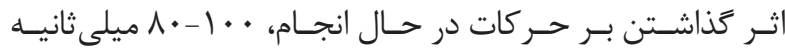

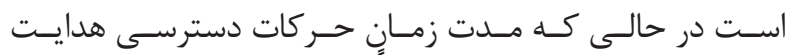

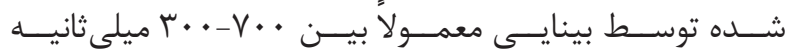

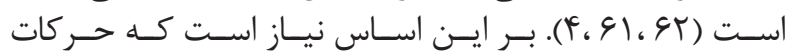

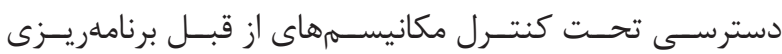

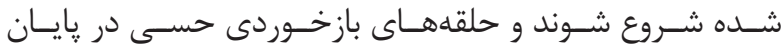

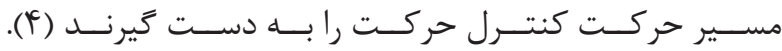

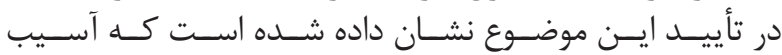

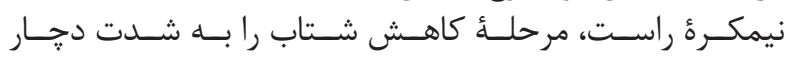

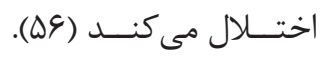

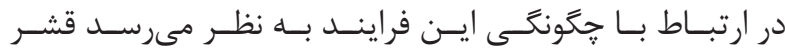

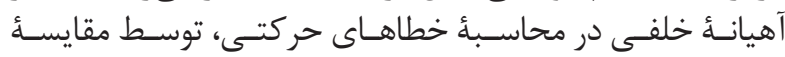

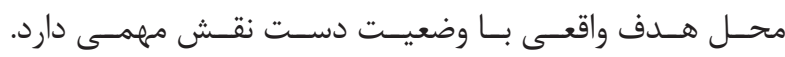

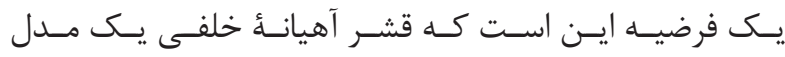

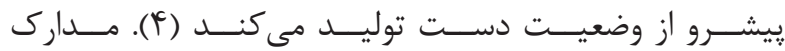

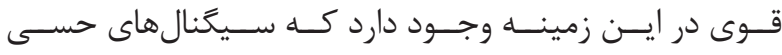

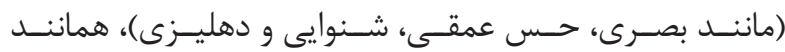

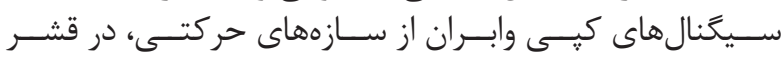

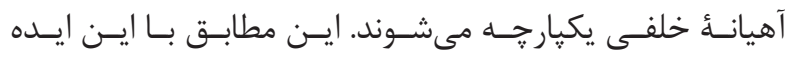

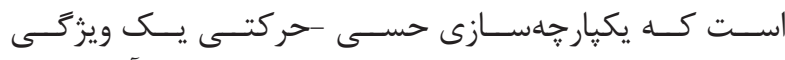

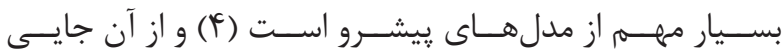

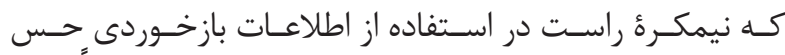

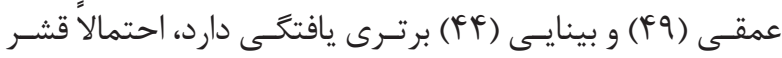

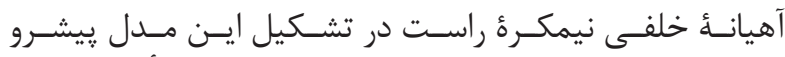

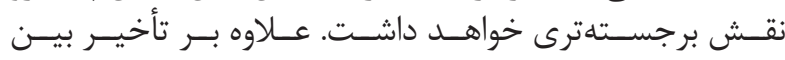

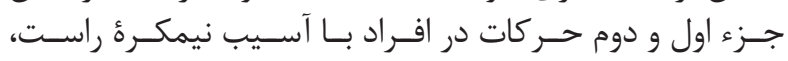




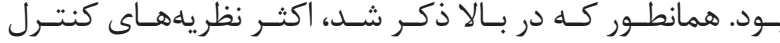

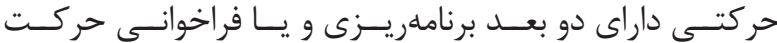

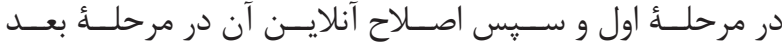

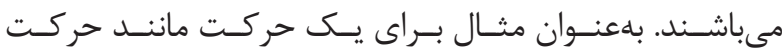

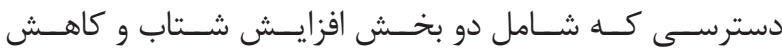

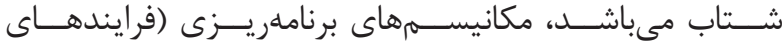

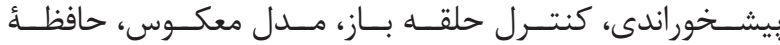

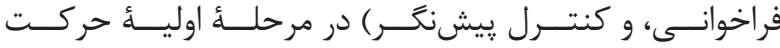

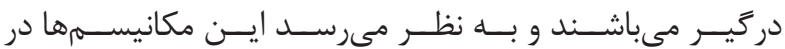

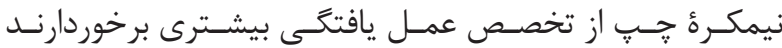

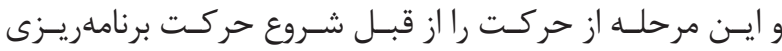

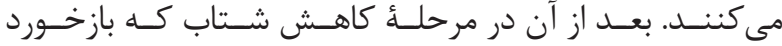

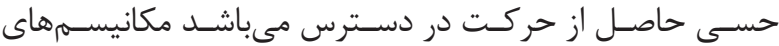

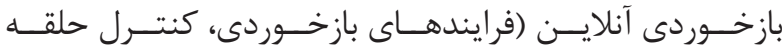

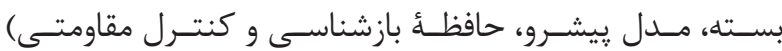

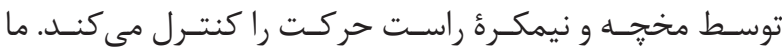

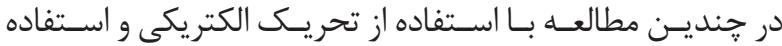

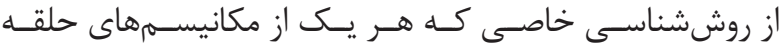

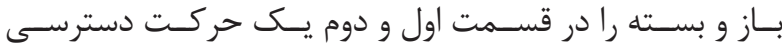

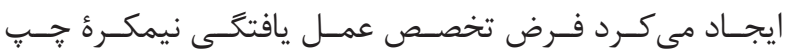

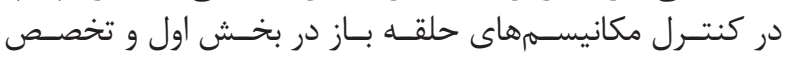

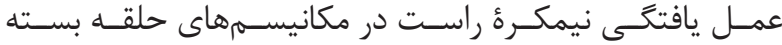

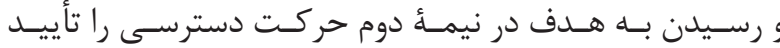

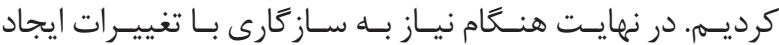

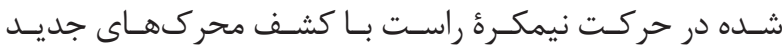

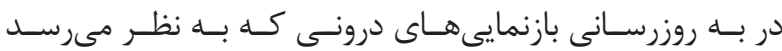

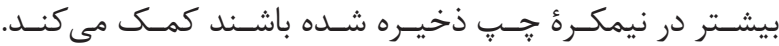

1. Yavari F, Towhidkhah F, Ahmadi-Pajouh MA. Are fast/slow process in motor adaptation and forward/ inverse internal model two sides of the same coin? Med Hypotheses. 2013; 81(4): 592-600.

2. Yadav V, Sainburg RL. Handedness can be explained by a serial hybrid control scheme. Neuroscience. 2014; 278: $385-96$.

3. Todorov E. Optimality principles in sensorimotor control. Nat Neurosci. 2004; 7(9): 907-15.

4. Desmurget M, Grafton S. Forward modeling allows feedback control for fast reaching movements. Trends Cogn Sci. 2000; 4(11): 423-31.

5. Milner TE. A model for the generation of movements requiring endpoint precision. Neuroscience. 1992; 49(2): 487-96.

6. Jeannerod M. The neural and behavioural organization of goal-directed movements: Oxford University Press. 1988. p. 296

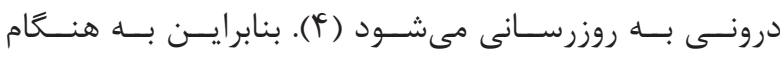

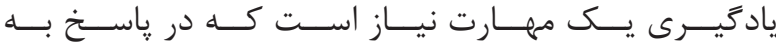

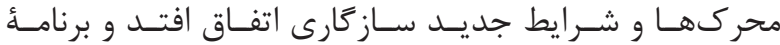

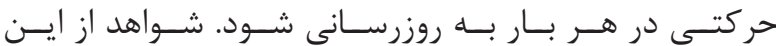

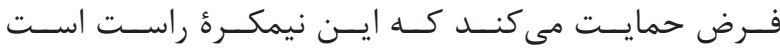

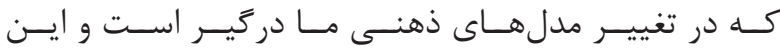

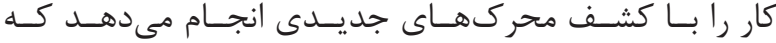

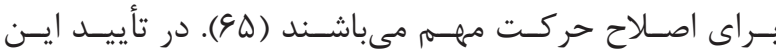

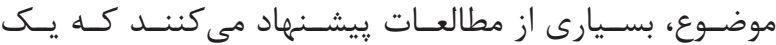

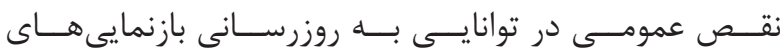

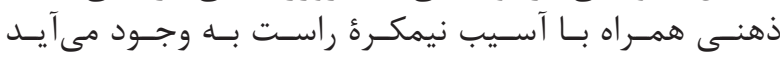

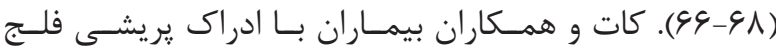

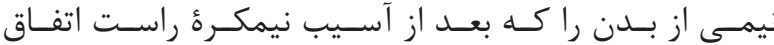

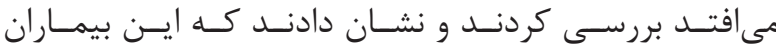

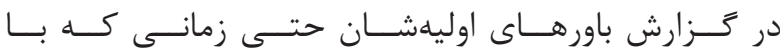

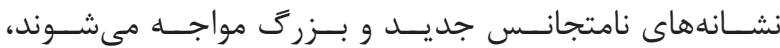

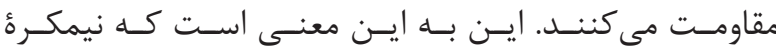

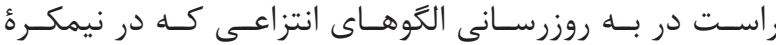

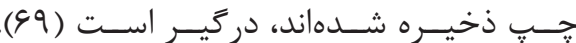

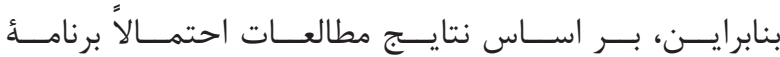

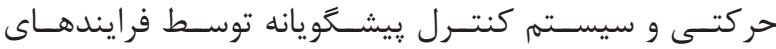

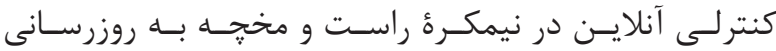

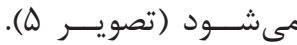

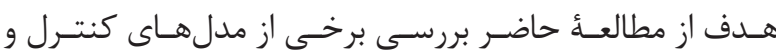

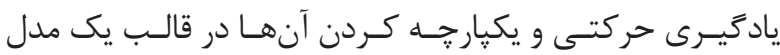

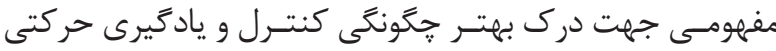

منابع

7. Carlton LG. Processing visual feedback information for movement control. J Exp Psychol Hum Percept Perform. 1981; 7(5): 1019-30.

8. Beaubaton D, Hay L. Contribution of visual information to feedforward and feedback processes in rapid pointing movements. Hum Mov Sci. 1986; 5(1): 19-34.

9. stroeve S. A learning feedback and feedforward neuromuscular control model for two degrees of freedom human arm movements. Hum Mov Sci. 1997; 16(5): 621-51.

10. Rosa M. Co-contraction role on human motor control. a neural basis. Journal of Novel Physiotherapies. 2015; 2015.

11. Bagesteiro LB, Sainburg RL. Nondominant arm advantages in load compensation during rapid elbow joint movements. J Neurophysiol. 2003; 90(3): 1503-13.

12. Haaland KY, Prestopnik JL, Knight RT, Lee RR. Hemispheric asymmetries for kinematic and positional aspects of reaching. Brain. 2004; 127(5): 1145-58. 


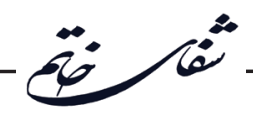

13. Jordan MI, Rumelhart DE. Forward models: Supervised learning with a distal teacher. Cogn Sci. 1992; 16(3): 307-54.

14. Mehta B, Schaal S. Forward models in visuomotor control. J Neurophysiol. 2002; 88(2): 942-53.

15. Kawato M. Internal models for motor control and trajectory planning. Curr Opin Neurobiol. 1999; 9(6): 718-27.

16. Tseng Y-w, Diedrichsen J, Krakauer JW, Shadmehr R, Bastian AJ. Sensory prediction errors drive cerebellumdependent adaptation of reaching. J Neurophysiol. 2007; 98(1): 54-62.

17. Porrill J, Dean P, Anderson SR. Adaptive filters and internal models: multilevel description of cerebellar function. Neural Netw. 2013; 47: 134-49.

18. Bastian AJ. Moving, sensing and learning with cerebellar damage. Curr Opin Neurobiol. 2011; 21(4): 596-601.

19. Cheng K-H. Adaptive fuzzy CMAC-based nonlinear control with dynamic memory architecture. J Franklin Inst. 2011; 348(9): 2480-502.

20. Max L, Guenther FH, Gracco VL, Ghosh SS, Wallace ME. Unstable or insufficiently activated internal models and feedback-biased motor control as sources of dysfluency: a theoretical model of stuttering. Contemp Issues Commun Sci Disord. 2004; 31: 105-22.

21. Dindo H, Zambuto D, Pezzulo G. Motor simulation via coupled internal models using sequential monte carlo. IJCAI Proceedings-International Joint Conference on Artificial Intelligence; 2011. 2113-9.

22. Wolpert DM, Diedrichsen J, Flanagan JR. Principles of sensorimotor learning. Nat Rev Neurosci. 2011; 12(12): 739-51.

23. Feys P, Helsen W, Nuttin B, Lavrysen A, Ketelaer $\mathrm{P}$, Swinnen $\mathrm{S}$, et al. Unsteady gaze fixation enhances the severity of MS intention tremor. Neurology. 2008; 70(2): 106-13.

24. Salmoni AW, Schmidt RA, Walter CB. Knowledge of results and motor learning: a review and critical reappraisal. Psychol Bull. 1984; 95(3): 355.

25. Schmidt RA, Lee TD. Motor learning and control. 1988.

26. Schmidt RA, White L. Evidence for an error detection mechanism in motor skills: a test of Adams' closed-loop theory. J Mot Behav. 1972: 4(3); 143-53.
27. Newell KM. Schema theory (1975): retrospectives and prospectives. Res Q Exerc Sport. 2003; 74(4): 383-8.

28. Sherwood D, Lee T. Cognitive effort and schema theory: Implications for a new theory of motor learning. Res Q Exerc Sport. 2003; 74: 376-82.

29. Schmidt RA. Motor control and learning . hu man kinetics Publishers. Inc. Champaign, Illinois. 1988: p. 105-16.

30. Schmidt RA, White JL. Evidence for an error detection mechanism in motor skills: a test of Adams' closed-loop theory. J Mot Behav. 1972; 4(3): 143-53.

31. Nicholson DE, Schmidt RA. Timing-task duration determines post-response error-detection capabilities. North American society for psychology of sport and physical activity annual meeting, Monterey, CA; 1991.

32. Coelho CJ, Przybyla A, Yadav V, Sainburg RL. Hemispheric differences in the control of limb dynamics: a link between arm performance asymmetries and arm selection patterns. J Neurophysiol. 2013; 109(3): 825-38.

33. Schaefer SY, Mutha PK, Haaland KY, Sainburg RL. Hemispheric specialization for movement control produces dissociable differences in online corrections after stroke. Cereb Cortex. 2012; 22(6): 1407-19.

34. Oliveira FT, Diedrichsen J, Verstynen T, Duque J, Ivry RB. Transcranial magnetic stimulation of posterior parietal cortex affects decisions of hand choice. PNAS. 2010; 107(41): 17751-6.

35. Teymuri M, Saberi Kakhki A, Taheri HR, Ghanaei CHamanabad A, Darainy M. The effect of brain hemisphere stimulation and how to specialize motor task programming: a transcranial direct current stimulation study. Journal of Neurological Sciences. 2017; 34(3).

36. Kheravi MT, Kakhki AS, Taheri H, Chamanabad AG, Darainy M. Researching hemispheric specialization in the control of final position accuracy using the transcranial direct current stimulation. Glob J Health Sci. 2017; 9(7): 138-49.

37. Teymuri M, Saberi Kakhki A, Taheri HR, Ghanaei CHamanabad A, Darainy M. The use of direct current s timulation to investigate the role of each hemisphere in motor learning of reaching task. Shefayekhatam. 2017; 5(4): 65-75.

38. Mutha PK, Sainburg RL, Haaland KY. Left parietal regions are critical for adaptive visuomotor control. J Neurosci. 2011; 31(19): 6972-81.

39. Zwinkels A, Geusgens C, Van de Sande P, 
Van Heugten C. Assessment of apraxia: inter-rater reliability of a new apraxia test, association between apraxia and other cognitive deficits and prevalence of apraxia in a rehabilitation setting. Clin Rehabil. 2004; 18(7): 819-27.

40. Schaefer SY, Haaland KY, Sainburg RL. Dissociation of initial trajectory and final position errors during visuomotor adaptation following unilateral stroke. Brain Res. 2009; 1298: 78-91.

41. Mutha PK, Haaland KY, Sainburg RL. The effects of brain lateralization on motor control and adaptation. J Mot Behav. 2012; 44(6): 455-69.

42. Stöckel T, Weigelt M. Brain lateralisation and motor learning: selective effects of dominant and non-dominant hand practice on the early acquisition of throwing skills. Laterality. 2012; 17(1): 18-37.

43. Schambra HM, Abe M, Luckenbaugh DA, Reis J, Krakauer JW, Cohen LG. Probing for hemispheric specialization for motor skill learning: a transcranial direct current stimulation study. J Neurophysiol. 2011; 106(2): 652-61.

44. Stöckel T, Wang J. Transfer of short-term motor learning across the lower limbs as a function of task conception and practice order. Brain Cogn. 2011; 77(2): 271-9.

45. Senff O, Weigelt M. Sequential effects after practice with the dominant and non-dominant hand on the acquisition of a sliding task in schoolchildren. Laterality. 2011; 16(2): 227-39.

46. Mutha PK, Sainburg RL, Haaland KY. Critical neural substrates for correcting unexpected trajectory errors and learning from them. Brain. 2011; 134(12): 3647-61.

47. Duff SV, Sainburg RL. Lateralization of motor adaptation reveals independence in control of trajectory and steady-state position. Exp Brain Res. 2007; 179(4): 551-61.

48. Schabowsky CN, Hidler JM, Lum PS. Greater reliance on impedance control in the nondominant arm compared with the dominant arm when adapting to a novel dynamic environment. Exp Brain Res. 2007; 182(4): 567-77.

49. Goble DJ, Brown SH. Upper limb asymmetries in the matching of proprioceptive versus visual targets. J Neurophysiol. 2008; 99(6): 3063-74.

50. Sainburg RL. Handedness: differential specializations for control of trajectory and position. Exerc Sport Sci
Rev. 2005; 33(4): 206-13.

51. Rosenbaum DA. Human motor control. $2^{\text {nd }}$ ed. Academic press. 2009. p. 528.

52. Schmidt R, Lee T. Motor Learning and performance, 5th ed with web study guide. Human Kinetics. 2014; 336.

53. Sainburg RL, Schaefer SY. Interlimb differences in control of movement extent. J Neurophysiol. 2004; 92(3): 1374-83.

54. Grafton ST, Desmurget M, Alexander G. Functional anatomy of error correction during pointing without vision of the hand. Ann Neurol 1999; 46(3): 447.

55. Inoue K, Kawashima R, Satoh K, Kinomura S, Goto R, Koyama M, et al. PET study of pointing with visual feedback of moving hands. J Neurophysiol. 1998; 79(1): 117-25.

56. Schaefer SY, Haaland KY, Sainburg RL. Ipsilesional motor deficits following stroke reflect hemispheric specializations for movement control. Brain. 2007; 130(8): 2146-58.

57. Anguera JA, Russell CA, Noll DC, Seidler RD. Neural correlates associated with intermanual transfer of sensorimotor adaptation. Brain Res. 2007; 1185: 136-51.

58. Serrien DJ, Ivry RB, Swinnen SP. Dynamics of hemispheric specialization and integration in the context of motor control. Nat Rev Neurosci. 2006; 7(2): 160-6.

59. Goldenberg G. Apraxia and beyond: life and work of Hugo Liepmann. Cortex. 2003; 39(3): 509-24.

60. Pearce J. Hugo Karl Liepmann and apraxia. Clin Med (Lond). 2009; 9(5): 466-70.

61. Hollerbach JM. Computers, brains, and the control of movement. Trends Neurosci. 1982; 5: 1-474.

62. Gerdes V, Happee R. The use of an internal representation in fast goal-directed movements: a modelling approach. Biol Cybern. 1994; 70(6): 513-24.

63. Diedrichsen J, White O, Newman D, Lally N. Usedependent and error-based learning of motor behaviors. J Neurosci. 2010; 30(15): 5159-66.

64. Verstynen T, Sabes PN. How each movement changes the next: an experimental and theoretical study of fast adaptive priors in reaching. J Neurosci. 2011; 31(27): 10050-9.

65. Bonato M. Neglect and extinction depend greatly on task demands: a review. Front Hum Neurosci. 2012; 6: 
195. doi: 10.3389/fnhum.2012.00195.

66. Stöttinger E, Filipowicz A, Marandi E, Quehl N, Danckert J, Anderson B. Statistical and perceptual updating: correlated impairments in right brain injury. Exp Brain Res. 2014; 232(6): 1971-87.

67. Shaqiri A, Anderson B. Priming and statistical learning in right brain damaged patients. Neuropsychologia. 2013;
51(13):2526-33.

68. Shaqiri A, Anderson B, Danckert J. Statistical learning as a tool for rehabilitation in spatial neglect. Front Hum Neurosci. 2013; 7: 224.

69. Vocat R, Saj A, Vuilleumier P. The riddle of anosognosia: does unawareness of hemiplegia involve a failure to update beliefs? Cortex. 2013; 49(7): 1771-81. 